Pacific Journal of Mathematic 


\title{
ON AUTOMORPHISMS OF SEPARABLE ALGEBRAS II
}

\author{
F. R. DEMEYeR
}

\begin{abstract}
A Galois theory for separable projective algebras over a class of commutative rings more general than Von Neumann regular rings with includes the full group of algebra automorphisms is presented. Fundamental facts concerning the group of units and the automorphisms of these algebras are also given.
\end{abstract}

A Galois theory for simple algebras over fields which included the full group of automorphisms was presented by G. Hochschile [5]. After the work of M. Auslander and O. Goldman [1] and S. Chase, D. K. Harrison, and A. Rosenberg [3] it was possible to generalize Hochschild's theory to semi-local rings with no idempotents other than 0 and 1 [4]. More recent results of $O$. Villamayor and D. Zelinsky [14], and $H$. Kreimer [8] permit the extension of Hochschild's theory to a much broader class of rings than in [4]. The context includes separable projective algebras over commutative regular rings and is stated explicitly at the beginning of $\S 3$. Theorem 3 and Theorem 5 of $\S 2$ have also been obtained by A. Magid and will appear in the Illinois Journal of Mathematics as part of an article entitled Pierce's representation and separable algebras.

We first state some of the results of [14] without proof. The Galois theory in [14] is then improved in a special case. Next, in $\S 2$, several basic properties of separable algebras are developed. The Galois theory which generalizes [4] is presented in $\S 3$. Henceforth $R$ always is a commutative ring with identity. All rings have identity, all subrings share the common identity, all modules are unitary, and all algebra homomorphisms carry identity to identity. Also $\otimes$ will mean $\otimes_{R}$. A familiarity with the basic properties of separable algebras is assumed. In particular, we employ a result of Villamayor [10] in the sequel which asserts that any separable projective $R$-algebra is finitely generated as an $R$-module.

1. We first enumerate, with corresponding nomenclature, several results in [14]. Let $B(R)$ be the Boolean algebra of idempotents of the commutative ring $R$. If $e, f \in B(R)$ the Boolean operations are given by $e \oplus f=e+f-e f$ and $e * f=e f$. Let spec $B(R)$ be the space of maximal (= prime) ideals in $B(R)$ and call the subsets $U_{e}$ of spec $B(R)$ defined for each $e \in B(R)$ by 


$$
U_{e}=\{p \in \operatorname{spec} B(R) \mid e \in p\}
$$

a base for the open sets of $\operatorname{spec} B(R)$. In this topology spec $B(R)$ is a totally disconnected compact Hausdorff space. If $p \in \operatorname{spec} B(R)$ we let $R_{p}=R / p \cdot R$, then $R_{p}$ is a homomorphic image of $R$. If $M$ is an $R$ module then $R_{p} \otimes M=M / p M$ is denoted $M_{p}$ and the natural image of $a \in M$ in $M_{p}$ is denoted $a_{p}$. In most of what follows we will use the compactness of spec $B(R)$ to recover information true at $R_{p}$ for each $p \in \operatorname{spec} B(R)$ to information about $R$.

The next seven results are proved in [14].

(2.7) If $M$ and $N$ are finitely generated projective $R$-modules the natural map from $\operatorname{Hom}_{R}(M, N)$ to $\operatorname{Hom}_{R_{p}}\left(M_{p}, N_{p}\right)$ denoted $f \rightarrow f_{p}$ is an isomorphism.

(2.9) If $a$ and $b$ are elements of the finitely generated $R$-module $M$ and $a_{p}=b_{p}$ for one $p \in \operatorname{spec} B(R)$ then $a_{q}=b_{q}$ for all $q$ in some neighborhood $U_{e}$ of $p$.

(2.11) If $N$ is a submodule of the finitely generated $R$-module $M$ and $N_{p}=M_{p}$ for all $p \in \operatorname{spec} B(R)\left(R_{p}\right.$ is flat over $R$ so $\left.N_{p} c M_{p}\right)$ then $N=M$.

(2.12) If $S$ is a finitely generated $R$-algebra and $u$ is an idempotent in $S_{p}$ for some $p \in \operatorname{spec} B(R)$, then there is an idempotent $v$ in $S$ such that $v_{p}=u$.

(2.13) $R_{p}$ has no idempotents other than 0 and 1 for each $p \in \operatorname{spec} B(R)$.

(2.14) Suppose $S$ is an $R$-algebra that is finitely presented as an $R$ module, let $F$ be a finite subset of $S$ and let $g$ be an $R_{p}$-algebra homomorphism of $S_{p}$ which is the identity on $F_{p}$. Then there is an $R$-algebra homomorphism $h$ of $S$ such that $h$ is the identity on $F$ and $h_{p}=g$.

(2.17) If $S$ is an $R$-algebra and $J$ is a finite group of $R$-algebra automorphisms of $S$; then for all $p \in \operatorname{spec} B(R),\left(S^{J}\right)_{p}=\left(S_{p}\right)_{p}^{J}$ where $S^{J}$ denotes the fixed ring under $J$.

Let $K$ be a commutative ring containing $R$ as a subring.

If $K$ is a projective separable $R$-algebra and if $K^{G}=R$ where $G$ is the group of all $R$-automorphisms of $K$, then $K$ is called a normal separable extension of $R$ with group $G$. If $H$ is a subset of $G$, the closure $\bar{H}$ of $H$ is the set of all automorphisms $g$ of $K$ such that for each $p \in \operatorname{spec} B(R)$ and each minimal idempotent $f_{p}$ in $K_{p}, f_{p} \cdot g_{p}=f_{p} \cdot h_{p}$ 
for some $h \in H$. A closed subgroup of $G$ is one equal to its own closure. Another result from [14] is

Lemma 1. Let $K$ be a normal separable extension of $R$ with group $G$, then the following statements are equivalent.

(1) For each $p \in \operatorname{spec} B(R), K_{p}$ is a normal separable extension of $R_{p}$ with group $G_{p}$.

(2) $\operatorname{Hom}_{R}(K, K)$ is generated as a $K$-module by R-algebra automorphism of $K$.

(3) $R$ is the fixed ring of some finite set (equivalently, finite group) of automorphisms of $S$.

An example in [14] shows that when $K$ is a normal separable $R$ algebra the equivalent conditions of Lemma 1 need not always hold. It is only in the situation where these conditions hold that the techniques we employ are effective. As a consequence of Lemma 1 we can restate Theorem 3.8 of [14].

THeorem 3.8. Let $K$ be a normal separable $R$ extension of $R$ with group $G$, if there is a finite subset $J$ of $G$ with $K^{J}=R$ then there is a one to one correspondence between the set of all separable subalgebras of $K$ and the set of all subgroups $H$ of $G$ which are the closure of some finite subgroup of $G$ by $H \rightarrow K^{H}$.

The result we require is in the situation where every idempotent in $K$ belongs to $R$. In this case Theorem 3.8 can be improved.

Corollary 2. Let $K$ be a normal separable extension of $R$ with group $G$. Assume $R$ is the fixed ring of a finite subset of $G$ and assume every idempotent in $K$ belongs to $R$. Then there is a one-toone correspondence between the separable subalgebras of $K$ containing $R$ and the closed subgroups $H$ of $G$ by $H \rightarrow K^{I I}$. The closed subgroup $H$ of $G$ is a normal subgroup of $G$ if and only if the corresponding subalgebra is a normal extension of $R$ with group $G / H$.

Proof. We apply Theorem 3.8 to give the subgroup-subring correspondence. Assume $H$ is a closed normal subgroup of $G$, then $G / H$ acts as a group of automorphisms of $K^{H}$ in the usual way and $\left(K^{H}\right)^{G / H}=R$. Let $\sigma$ be an $R$-automorphism of $K^{H}$. By hypothesis on the idempotents in $K$ and Theorem 3.5 of [3] we have for each $p \in \operatorname{spec} B(R)$ an automorphism $\tau_{p}$ of $K_{p}$ which extends $\sigma_{p}$ on $K_{p}^{H p}$. Use (2.14) to lift $\tau_{p}$ to an automorphism $\tau$ on $K$. Since $K^{H}$ is finitely generated and projective over $R$ (Proposition 1.5 of [4]), $\operatorname{Hom}_{R}\left(K^{I I}, K\right.$ ) is finitely generated over $R$ so there is by (2.9) and (2.7) a neighborhood 
$U$ of $p$ such that for each $q \in U, \tau_{q}$ restricted to $\left(N^{H}\right)_{q}$ is $\sigma_{q}$. Cover spec $B(R)$ with such neighborhoods and use compactness of spec $B(R)$ to obtain a decomposition $R=R e_{1} \oplus \cdots \oplus R e_{n}$ and automorphisms $\tau_{i}$ of $K e_{i}$ with $\tau_{i}$ restricted to $\left(K^{H}\right) e_{i}$ equal to $\sigma$ on $\left(K^{H}\right) e_{i}$ (since $e_{i} \in R$, $\left.\sigma\left(e_{i}\right)=\tau\left(e_{i}\right)=e_{i}\right)$. The automorphism $\tau$ on $K$ constructed from the $\tau_{i}$ extends $\sigma$ and is in $G$ so $K^{H}$ is a normal separable extension of $R$ with group $G / H$. Conversely, assume $H$ is a closed subgroup of $G$ and $K^{H}$ is a normal separable extension of $R$. Then by Theorem 2.3 of [3] and (2.17) we know that for each $p \in \operatorname{spec} B(R)$ and each $\sigma \in G$

$$
\sigma_{p}\left(K^{H}\right)_{p}=\sigma_{p}\left(K_{p}^{H p}\right)=K_{p}^{H p}=\left(K^{H}\right)_{p} .
$$

Since $K^{I I}$ is finitely generated over $R$, (2.7) implies $\sigma\left(K^{H}\right)=K^{H}$. This is equivalent to the statement that $H$ is a normal subgroup of $G$ and completely proves the corollary.

With the hypothesis of Corollary 2, if $N$ is a normal separable extension of $R$ in $K$ which corresponds to the closed normal subgroup $H$ of $G$ observe that there is a finite subset of $G / H$ on $N$ leaving exactly $R$ fixed. Therefore our Galois theory also applies to the extension $N$ of $R$ with group $G / H$.

2. We now derive some properties of separable algebras. The group of isomorphism classes of rank $=1$ projective $R$-modules forms a group called the class group (Picard group) of $R$. Generalizing Theorem 3.6 of [1] we have

Theorem 3. Let $A$ be a central separable $R$-algebra. Assume the class group of $R_{p}$ is trivial for each $p \in \operatorname{spec} B(R)$, then every $R$-algebra automorphism of $A$ is an inner automorphism.

Proof. Let $\sigma$ be an algebra automorphism of $A$. By Theorem 3.6 of [1] we know $\sigma_{p}$ induces an inner automorphism of $A_{p}$ for each $p \in \operatorname{spec} B(R)$. Let $\sigma_{p}=i_{\bar{x}}$ on $A_{p}$, then there exists a $\bar{y} \in A_{p}$ with $\bar{x} \bar{y}=1$. Lift $\bar{x}$ and $\bar{y}$ to elements $x^{\prime}$ and $y^{\prime}$ in $A$. By (2.9) there is a neighborhood $U_{e}$ of $p$ so that for all $q \in U_{e}$ we have $x_{q}^{\prime} y_{q}^{\prime}=1$. Therefore $\left(x^{\prime} e\right)\left(y^{\prime} e\right)=e$ and $\left(x^{\prime} e\right)_{p}=\bar{x},\left(y^{\prime} e\right)_{p}=\bar{y}$. Let $x=(1-e)+x^{\prime} e$ and $y=(1-e)+y^{\prime} e$. Then $x, y \in A$ and $x y=1$. Moreover $i_{x}$ is an inner automorphism of $A$ with $\left(i_{x}\right)_{p}=i_{\bar{x}}$. Now $\operatorname{Hom}_{R}(A, A)$ is a finitely generated projective $R$-module so by (2.9) there is a neighborhood $U$ of $p$ in spec $B(R)$ with $\left(i_{x}\right)_{q}=\sigma_{q}$ for all $q \in U$. Cover spec $B(R)$ with such neighborhoods, since spec $B(R)$ is compact one can find a finite subcover. Thus $R=R e_{1} \oplus \cdots \oplus R e_{n}$ with $e_{i}^{2}=e_{i} \in R$ and there are elements $x_{j} \in A e_{j}(j=1, \cdots n)$ such that $x_{j}$ is a unit in $A e_{j}$ and $\sigma$ on $A e_{j}$ is inner automorphism by $x_{j}$. Let $x=x_{1}+\cdots+x_{n}$, then $\sigma$ is inner automorphism by $x$ on $A$. 
A corollory of this result shows that we are in the context of [9].

CoRollary 4. Let $A$ be a separable projective R-algebra with center $K$, and assume the class group of $K_{p}$ is trivial for each $p \in \operatorname{spec} B(R)$, then every $R$-algebra automorphism of $A$ which is not inner is represented nontrivially on $K$.

Proof. For $p \in \operatorname{spec} B(R), K_{p}$ is a finitely generated projective separable $R_{p}$-algebra and $R_{p}$ has no idempotents other than 0 and 1 . Thus $C_{p}$ is a finite direct sum of finitely generated projective separable $R_{p}$-algebras whose only idempotents are 0 and 1 . The class group of $K_{p}$ is the product of the class groups of the summands and the summands correspond to the elements of spec $B(K)$ so Theorem 3 applies.

Our inability to extend the next result restricts the generality of the Galois theory in $\S 3$. See the examples following Theorem 1.1 and Theorem 1.2 of [4].

THEOREM 5. Let $R$ be a commutative ring, assume for each $p \in \operatorname{spec} B(R)$ that $R_{p}$ is a semi-local ring, (finite number of maximal ideals). Let $A$ be a separable projective $R$-algebra with center $K$ and let $B$ be a separable $R$-subalgebra of $A$ with center $C$ containing $K$. Assume every idempotent in $C$ belongs to $R$. Let $\sigma$ be an $R$-algebra monomorphism from $B$ into $A$ leaving $K$ fixed, then $\sigma$ can be extended to an inner automorphism of $A$.

Proof. We know for any $p \in \operatorname{spec} B(R)$ that $A_{p}$ is a projective separable $R_{p}$ algebra with center $K_{p}$ and $B_{p}$ is a separable subalgebra with center $C_{p}$ containing $K_{p}$. By hypothesis on the idempotents in $C$ it follows that the only idempotents in $C_{p}$ are 0 and 1 . By the lemma on page 25 of [4], $B$ is projective over $R$ so $\operatorname{Hom}_{R}(B, A)$ is a finitely generated projective $R$-module and (2.7) implies $\operatorname{Hom}_{R}(B, A)_{p}=\operatorname{Hom}_{R_{p}}\left(B_{p}, A_{p}\right)$. With this identification, Theorem 1.2 of [4] gives an inner automorophism $i_{\bar{x}}$ on $A_{p}$ which extends $\sigma_{p}$ on $B_{p}$. As in the proof of Theorem 3 lift $\bar{x}$ to a unit $x$ in $A$ so that $i_{x}$ is an inner automorphism of $A$ with $\left(i_{x}\right)_{p}=i_{\bar{x}}$. Apply (2.9) to get a neighborhood $U_{e}$ of $p$ so that for all $q \in U_{e}, \sigma_{q}=\left(i_{x}\right)_{q}$ on $B_{q}$. Applying the same compactness argument as used in Theorem 3 we construct an inner automorphism $i_{y}$ on $A$ which extends $\sigma$.

If $R$ is a regular ring (in the sense of Von Neumann) then $R$ satisfies the hypothesis of Theorem 5 since every principal ideal in a regular ring is generated by an idempotent. Thus if $R$ is regular, $R_{p}$ is a field for each $p \in \operatorname{spec} B(R)$. The next result improves Theorem 


\section{5 of $[4]$.}

THEOREM 6. Let $A$ be a separable projective algebra over $K$ and let $J$ be a finite group of ring automorphisms of $A$ with the property that $J$ restricted to $K$ is a group of automorphisms of $K$ isomorphic to J. Let $R=K^{J}$ and assume $K$ is a separable finitely generated $R$-algebra with every idempotent in $K$ belonging to $R$. If $B=A^{J}$, then $B$ is a separable projective $R$-algebra and $A=B \otimes K$.

Proof. The hypothesis of Theorem 1.5 in [4] is satisfied at each $p \in \operatorname{spec} B(R)$. Therefore the natural homomorphism from $B \otimes K$ to $A$ is an isomorphism at each $p \in \operatorname{spec} B(R)$. Applying (2.7) and (2.11) we conclude $B \otimes K=A$. Now $K$ is a finitely generated, projective, separable $R$-algebra so $R \cdot 1$ is an $R$-direct summand of $K$ (see Proposition A.4 of [1]). By ([2], IX, 9.1) $B$ is separable over $R$. By the lemma on page 2.5 of [4] $B$ is projective over $R$.

The next result includes a correction of Lemma 1.8 in [4]. Let $Z$ be the ring of integers.

Lemma 7. Let $A$ be a separable projective $R$-algebra, then $A$ is generated as an algebra by its units if and only if $A_{p}$ is generated as an $R_{p}$ algebra by its units for all $p \in \operatorname{spec} B(R)$. Moreover, if $R_{p}$ is semi-local then $A_{p}$ is not generated as an algebra by its units if and only if $R_{p} / \operatorname{Rad}\left(R_{p}\right)=Z /(2) \oplus R^{\prime}$ and the central component of $A_{p} / \operatorname{Rad}\left(A_{p}\right)$ over $Z /(2)$ contains a direct factor isomorphic to $Z /(2) \oplus Z /(2)$.

Proof. Assume that $A_{p}$ is generated by its units for all $p \in \operatorname{spec} B(R)$. As in the proof of Theorem 3, each unit in $A_{p}$ lifts to a unit in $A$. Thus if $N$ is the $R$-subalgebra of $A$ generated by the units of $A$ we know $N_{p}=A_{p}$ for all $p \in \operatorname{spec} B(R)$. By (2.11) this implies $N=A$. The converse is clear.

For the last statement observe that the proof of Lemma 1.8 in [4] implies $A_{p}$ is generated by its units over $R_{p}$ if and only if $A_{p} / \operatorname{Rad}\left(A_{p}\right)$ is generated by its units over $R_{p} / \operatorname{Rad}\left(R_{p}\right)$. Now $R_{p} / \operatorname{Rad}\left(R_{p}\right)$ is a finite direct sum of fields and $A_{p} / \operatorname{Rad}\left(A_{p}\right)$ is a finite direct sum of semi-simple algebras over fields. It is an elementary exercise to show that the only semi-simple algebras over a field not generated by units occurs when the algebra contains $Z /(2) \oplus Z /(2)$ as a direct summand over the field $Z /(2)$. This proves the lemma.

More information about automorphisms of separable algebras is contained in 
Lemma 8. Let $A$ be a projective separable $R$-algebra with center $K$ and assume every idempotent in $K$ belongs to $R$, then any $R$ homomorphism $\sigma$ from $A$ to $A$ is an automorphism of $A$.

Proof. Observe that $\sigma$ is an $R$-homomorphism from $K$ to $K$. Consider the exact sequence

$$
0 \longrightarrow \operatorname{ker}(\sigma) \longrightarrow K \stackrel{\sigma}{\longrightarrow} \sigma(K) \longrightarrow 0 \text {. }
$$

Now $\sigma(K)$ is a separable $R$-subalgebra of $K$ so by the lemma on page 25 of [4] $\sigma(K)$ is projective over $R$. Therefore $\operatorname{ker}(\sigma)$ is an ideal direct summand and is generated by an idempotent in $K$. But every idempotent in $K$ belongs to $R$ and $\sigma$ is one-to-one on $R$ so $\sigma$ is oneto-one on $K$. For each $p \in \operatorname{spec} B(R)$ we know $\sigma_{p}: K_{p} \rightarrow K_{p}$ is one-toone. $K_{p}$ has no idempotents other than 0 and 1 and $K_{p}$ can be imbedded in a Galois (in the sense of [3]) extension of $R_{p}$ by [6] so Theorem 2.3 of [3] and the fact that $\operatorname{Rank}_{R_{p}}\left(K_{p}\right)=\operatorname{Rank}_{R_{p}}\left(\sigma_{p}\left(K_{p}\right)\right)$ implies $\sigma_{p}$ is also onto $K_{p}$. By (2.11) and (2.7) this implies $\sigma(K)=K$. Since there is a one-to-one correspondence between the two sided ideals of $A$ and those of $K$ by $a c K$ corresponds to $A a$ we know $\operatorname{ker}(\sigma)=0$ on $A$ and $\sigma$ is one to one on $A$.

Now $\sigma(A)$ is a central separable $K$-algebra (since $\sigma(K)=K$ ) and $\operatorname{Rank}_{K}(A)=\operatorname{Rank}_{K}(\sigma(A))$ since $\sigma(A)$ is one to one. By Theorem 3.3 of [1], $A=\sigma(A) \bigotimes_{K} A^{\prime}$ where $A^{\prime}$ is a central separable $K$-algebra. By a rank argument, $A^{\prime}=K$ and $\sigma$ maps $A$ onto $A$. This proves the lemma.

Lemma 9. Let $K$ and $L$ be commutative separable $R$-subalgebras of the finitely generated projective $R$-algebra $A$, then $K \cap L$ and $K \cdot L$ are separable projective $R$-subalgebras of $A$.

Proof. First, $K \cdot L$ is a homomorphic image of $K \otimes L$ and therefore is separable over $R$. By the lemma on page 25 of [4] $K \cdot L$ is projective over $R$. To prove $K \cap L$ is separable over $R$ we employ the construction in $\S 3$ of [12] to find a commutative projective separable $R$-algebra $N$ containing $K \cdot L$ such that there is a finite group $J$ of automorphisms of $N$ with $N^{J}=R$. By Theorem 3.8 there are finite sets $H, H^{\prime}$ of $R$-automorphisms of $N$ with $N^{H}=K$ and $N^{I I^{\prime}}=L$. Observe that $N^{I I \cdot I I^{\prime}}=K \cap L$ which by Theorem 3.8 implies $K \cap L$ is projective and separable over $R$.

3. Throughout this section we shall adhere to the following notations and assumptions. $R$ is a commutative ring with $R_{p}$ with semilocal for each $p \in$ spec $B(R) . \quad A$ is a separable projective $R$-algebra with 
center $K$, and every idempotent of $K$ belongs to $R$. Let $G$ be the group of all $R$-algebra automorphisms of $A$ and assume $A^{G}=R$. Also we assume there is a finite subset of $G$ whose restrictions give a finite set $J$ of automorphisms of $K$ with $K^{J}=R$.

If $H$ is a subgroup of $G$ we let $H_{0}$ be the normal subgroup of $H$ consisting of those elements of $H$ which are inner automorphisms of $A$. We let $R(H)$ be the subring of $A$ generated as a $K$-algebra by all the units giving the inner automorphisms in $H$. If $B$ is an $R$ subalgebra of $A$ we let $G_{B}$ be the group of all elements in $G$ leaving $B$ elementwise fixed.

Call a subgroup $H$ of $G$ complete if every inner automorphism of $A$ by an element of $R(H)$ is in $H$ and if when restricted to $K$, every element of $G / H_{0}$ which leaves $K^{H}$ fixed is in $H / H_{0}$. We show later that this last condition implies that on $K$ we have $H$ a closed group of automorphisms in the sense defined in $\S 1$. Call a subgroup $H$ of $G$ regular if $H$ is complete, $R(H)$ is a separable $K$-algebra, and every central idempotent in $R(H)$ belongs to $K$.

If $B$ is an $R$-algebra of $A$ we call $B$ regular if $B$ is separable as an $R$-algebra, if every idempotent in the center of $B \bigotimes_{B \cap K} K$ is in $K$, and if the commutator of $K \cdot B$ in $A$ is generated as a $K$-algebra by its units.

If $R$ is a semi-local ring whose only idempotents are 0 and 1 these definitions reduce to [4]. For the same reasons as in the Galois theory for simple algebras over fields, it is the regular subgroups and the regular subalgebras which the Galois theory relates.

THEOREM 10. If $H$ is a regular subgroup of $G$, then $A^{H}$ is a regular $R$-subalgebra of $A$, and $H$ is the group of all automorphisms of $A$ leaving $A^{H}$ fixed.

Proof. The commutator of $R(H)$ in $A$ is $A^{H_{0}}$; thus by Theorem 2 of [7], $A^{H_{0}}$ is a separable $R$-algebra. Now $H$ leaves $R(H)$ setwise invariant so $H$ leaves $A^{H_{0}}$ setwise invariant. Restricting $H$ to $A^{H_{0}}$ yields a group of automorphisms $H^{\prime}$ of $A^{H_{0}}$ leaving $A^{H}$ fixed which is isomorphic to $H / H_{0}$. Similarly $H$ leaves $K$ setwise invariant. Since all automorphisms of $A$ leaving $K$ fixed are inner by Theorem $3, H$ restricted to $K$ is also isomorphic to $H / H_{0}$ and can be viewed as the restriction of $H^{\prime}$ to $K$. By our assumptions there is a finite subset $J$ of $H^{\prime}$ with $K^{J}=K^{H^{\prime}}=K \cap A^{H}$. By Corollary $2, K \cap H^{H}$ is separable over $R$. The center of $A^{H_{0}}$, being the same as the center of $R(H)$, is projective and separable over $K$. Furthermore $K$ is projective and separable over $R$ so $A^{H_{0}}$ is projective and separable over $R$. By Theorem 6 we know $\left(A^{H_{0}}\right)^{J}=B$ is separable over $K^{J}$ and $A^{H_{0}}=B \otimes K$ 
(tensor over $K^{J}$ ). Since $K^{H}$ is separable over $R, B$ is separable over $R$. Every idempotent in the center of $A^{H_{0}}$ is in $K$ by assumption on $R(H)$. Now $A^{H} c B$ but if $\sigma \in H$ and $\left.\sigma\right|_{B} \neq e$ then $\sigma \otimes 1$ is an automorphism of $B \otimes K=A^{H_{0}}$ which is the identity on $K$ so $\sigma \otimes 1$ extends by Theorem 3 to an element of $H_{0}$. This is impossible since $B c A^{H_{0}}$. Thus $A^{H}=B$ is a regular subalgebra of $A$.

To prove the last statement of the theorem observe that $H_{0}$ contains all the inner automorphisms leaving $A^{H}$ fixed. Also $H^{\prime}=H / H_{0}$ contains all the automorphisms of $A^{H_{0}}$ leaving $A^{H}$ fixed since $A^{H_{0}}=$ $A^{H} \otimes K$ (tensor over $\left.A^{H} \cap K\right)$. Moreover $H / H_{0}$ is closed when viewed as a group of automorphisms of $K$.

TheOREM 11. Let $B$ be a regular subalgebra of $A$ containing $R$, then the group $G_{B}$ is a regular subgroup of $B$ and $B$ is the fixed ring of $G_{B}$.

Proof. If $C$ is the center of $B$, then $C \otimes_{B \cap K} K$ is the center of $B \otimes_{B \cap K} K$, so $C \cdot K$ is the center of $B \cdot K$ and is separable over $R$. By Lemma $9 C \cap K$ is separable over $R$, therefore $B \cap K$ is separable over $R$. By our assumption there is a finite subset of $G$ whose restrictions to $K$ leave exactly $R$ elementwise fixed so by Theorem 6 we can write $A=A^{a} \otimes K$. By Corollary 2 there is a finite subset $J^{\prime}$ of the $R$ automorphism group of $K$ with $K^{J^{\prime}}=B \cap K$. The representation of $A$ as $A^{\theta} \otimes K$ insures that every element of $J^{\prime}$ extends to an element in $G / G_{0}$. Next we show the natural map $j: B \otimes_{B \cap K} K \rightarrow B \cdot K$ is an isomorphism. Now ker $j=I \cdot\left(B \otimes_{B \cap K} K\right)$ where $I$ is the kernel of the restriction of $j$ to $C \otimes_{C \cap K} K$. Consider the exact sequence of $C \otimes_{c \cap K} K$ modules

$$
0 \longrightarrow I \longrightarrow C \otimes_{C \cap K} K \longrightarrow C \cdot K \longrightarrow 0 \text {. }
$$

Now $Z \cdot K$ is a projective $R$-module, so since $C \otimes_{c n_{K}} K$ is a separable $R$-algebra $Z \cdot K$ is a projective $C \otimes_{C \cap K} K$-module. Hence the sequence splits and $I$ is generated by an idempotent. Since every idempotent in $C \otimes_{C \cap K} K$ belongs to $K, I=0$ and $j$ is an isomorphism.

Extend each $\sigma^{\prime}$ in $J^{\prime}$ to $B \cdot K=B \otimes_{B \cap K} K$ by

$$
\sigma^{\prime}(b k)=b \sigma^{\prime}(k) \text {. }
$$

Lift $\sigma^{\prime}$, viewed as an element of $G / G_{0}$, to an element $\sigma$ of $G$. Then $\sigma$ restricted to $K$ is $\sigma^{\prime}$ so $\sigma^{-1} \sigma^{\prime}: B \cdot K \rightarrow A$ leaves $K$ fixed. Since every central idempotent in $B \cdot K$ belongs to $K, \sigma^{-1} \sigma^{\prime}$ extends to an inner automorphism $\tau$ of $A$ by Theorem 5. Now $\sigma^{\prime}$ is $\sigma \tau$ restricted to $B \cdot K$ so $\sigma^{\prime}$ extends to an automorphism of $A$. Each $\sigma^{\prime}$ in $J^{\prime}$ in this way extends to an element of $G_{B}$, and since $J^{\prime}$ defined on $B \cdot K$ leaves ex- 
actly $B$ fixed, $G_{B}$ must leave exactly $B$ fixed.

The commutator $L$ in $A$ of $B$ is the commutator in $A$ of $B \cdot K$, so is finitely generated and separable over $K$ with every central idempotent of $L$ in $K$. Clearly, any automorphism of $G_{B}$ comes from a unit in $L$ and every unit in $L$ defines an inner automorphism in $G_{B}$. To show that $L=R\left(G_{B}\right)$ and consequently that $G_{B}$ is regular, it suffices to show that $L$ is generated as a $K$-algebra by its units. This follows from the definition of regularity for $B$.

Retain the notation of Theorem 11 in Theorem 12.

THEOREM 12. If $B$ is a regular subalgebra of $A$ and $G_{B}$ is a normal subgroup of $G$, then $G / G_{B}$ is the group of all algebra automorphisms of $B$.

Proof. Set $H=G_{B}$. The restriction map from $G$ to the automorphism group of $B$ has kernal $H$. Since $H$ is normal in $G$, this implies every element in $G / H$ can be viewed as an automorphism of $B$. The proof will be complete when we extend any $R$-automorphism $\sigma^{\prime}$ on $B$ to an automorphism of $A$. Now $H^{\prime}=H / H_{0}$ is a normal subgroup of $G^{\prime}=G / G_{0}$ by the second isomorphism theorem so restricting our attention to $K$ we know by Corollary 2 that $B \cap K$ is a normal separable extension of $R$ with group $G^{\prime} / H^{\prime}$ and $G^{\prime} / H^{\prime}$ contains all automorphisms of $B \cap K$ leaving $R$ fixed. View $\sigma^{\prime}$ as an element of $G^{\prime} / H^{\prime}$ and extend to an element $\sigma$ of $G$. Then $\tau^{\prime}=\sigma^{-1} \sigma^{\prime}$ is an automorphism of $B$ leaving $B \cap K$ fixed. Define $\tau^{\prime}$ on $B \otimes_{B \cap K} K=B \cdot K$ by $\tau^{\prime}(b k)=\tau^{\prime}(b) k$; then $\tau^{\prime}$ is an automorphism of $B \cdot K$ leaving $K$ fixed. By Theorem 5, $\tau^{\prime}$ extends to an automorphism $\tau$ of $G$. For any $b \in B$, $\sigma \tau(b)=\sigma \tau^{\prime}(b)=\sigma\left(\sigma^{-1} \sigma\right)(b)=\sigma^{\prime}(b)$ so $\sigma \tau$ is the desired automorphism.

Following [5] we call a subalgebra $B$ of $A$ almost regular in case $B$ is separable over $R$, every central idempotent in $B \cdot K$ belongs to $K$, and the commutator of $B \cdot K$ in $A$ is generated by its units. Every regular algebra is almost regular and in this context the converse also holds.

Theorem 14. With $A, K, R$, and $G$ as above, every almost regular $R$-subalgebra of $A$ is regular.

Proof. Let $B$ be an almost regular $R$-subalgebra of $A$. Since every idempotent in $B \cdot K$ belongs to $K$ it suffices to show that the natural map

$$
j: B \otimes_{B \cap K} K \longrightarrow B \cdot K
$$

is an isomorphism. As in the proof of Theorem 11 we can restrict our 
attention to the natural map $j: C \otimes_{C \cap K} K \rightarrow C \cdot K$ where $C$ is the center of $B$.

Since $C \cap K=L$ is separable over $R$ by Lemma 9 and projective by the lemma on page 25 of [4] both $C$ and $K$ are projective when viewed as $L$-algebras. Thus $C \bigotimes_{L} K=C \cdot K \oplus \operatorname{ker}(j)$. By Theorem 2.3 of [4], $\operatorname{ker}(j)=0$ for every $p \in \operatorname{spec} B(R)$ so $\operatorname{ker}(j)=0$ and $j$ is an isomorphism.

\section{BIBLIOGRAPHY}

1. M. Auslander and O. Goldman, The Brauer group of a commutative ring, Trans. Amer. Math. Soc. 97 (1960), 367-409.

2. H. Cartan and S. Eilenberg, Homological algebra, Princeton Univ. Press, Princeton, N. J., 1956.

3. S. Chase, D. K. Harrison, and A. Rosenberg, Galois theory and cohomology of commutative rings, Mem. Amer. Math. Soc. 52 (1965).

4. L. N. Childs and F. R. DeMeyer, On automorphisms of separable algebras, Pacific J. Math. 23 (1967), 25-34.

5. G. Hochschild, Automorphisms of simple algebras, Trans. Amer. Math. Soc. 69 (1950), 292-301.

6. G. J. Janusz, Separable algebras over commutative rings, Trans. Amer. Math. Soc. 122 (1966), 461-479.

7. T. Kanzaki, On commutor rings and the Galois theory of separable algebras, Osaka J. Math. 1 (1964), 103-115.

8. H. F. Kreimer, A note on the outer Galois theory of rings, Pacific J. Math. (to appear)

9. - Outer Galois theory for separable algebras. (to appear)

10. T. Nagahara, A note on Galois theory of commutative rings, Proc. Amer. Math. Soc. 18 (1967), 334-340.

11. R. S. Pierce, Modules over commutative regular rings, Mem. Amer. Math. Soc. 70 (1967).

12. O. E. Villamayor, Separable algebras and Galois extensions, Osaka J. Math. 4 (1967), 161-171.

13. O. E. Villamayor and D. Zelinsky, Galois theory for rings with finitely many idempotents, Nagoya Math. J. 27 (1966) 721-731.

14. - Galois theory with infinitely many idempotents, Nagoya Math. J. 35 (1969), 83-98.

Received April 9, 1969 



\title{
PACIFIC JOURNAL OF MATHEMATICS
}

\author{
EDITORS
}

H. SAMELSON

Stanford University

Stanford, California 94305

J. DugunduI

Department of Mathematics

University of Southern California

Los Angeles, California 90007

RICHARD PIERCE

University of Washington

BASIL GORDON*

University of California

Seattle, Washington 98105

Los Angeles, California 90024

\section{ASSOCIATE EDITORS}

E. F. BeCKenbaCh

B. H. NeumanN

F. WOLF

K. YosHIDA

\section{SUPPORTING INSTITUTIONS}

\author{
UNIVERSITY OF BRITISH COLUMBIA \\ CALIFORNIA INSTITUTE OF TECHNOLOGY \\ UNIVERSITY OF CALIFORNIA \\ MONTANA STATE UNIVERSITY \\ UNIVERSITY OF NEVADA \\ NEW MEXICO STATE UNIVERSITY \\ OREGON STATE UNIVERSITY \\ UNIVERSITY OF OREGON \\ OSAKA UNIVERSITY \\ UNIVERSITY OF SOUTHERN CALIFORNIA
}

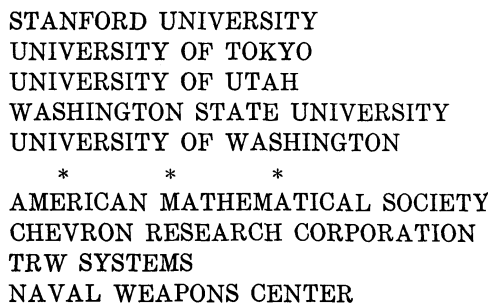

The Supporting Institutions listed above contribute to the cost of publication of this Journal, but they are not owners or publishers and have no responsibility for its content or policies.

Mathematical papers intended for publication in the Pacific Journal of Mathematics should be in typed form or offset-reproduced, double spaced with large margins. Underline Greek letters in red, German in green, and script in blue. The first paragraph or two must be capable of being used separately as a synopsis of the entire paper. It should not contain references to the bibliography. Manuscripts, in duplicate if possible, may be sent to any one of the four editors. Please classify according to the scheme of Math. Rev. 36, 1539-1546. All other communications to the editors should be addressed to the managing editor, Richard Arens, University of California, Los Angeles, California, 90024.

50 reprints are provided free for each article; additional copies may be obtained at cost in multiples of 50 .

The Pacific Journal of Mathematics is published monthly. Effective with Volume 16 the price per volume (3 numbers) is $\$ 8.00$; single issues, $\$ 3.00$. Special price for current issues to individual faculty members of supporting institutions and to individual members of the American Mathematical Society: $\$ 4.00$ per volume; single issues $\$ 1.50$. Back numbers are available.

Subscriptions, orders for back numbers, and changes of address should be sent to Pacific Journal of Mathematics, 103 Highland Boulevard, Berkeley, California, 94708.

PUBLISHED BY PACIFIC JOURNAL OF MATHEMATICS, A NON-PROFIT CORPORATION

Printed at Kokusai Bunken Insatsusha (International Academic Printing Co., Ltd.), 7-17, Fujimi 2-chome, Chiyoda-ku, Tokyo, Japan.

* Acting Managing Editor. 


\section{Pacific Journal of Mathematics}

\section{Vol. 32, No. 3 \\ March, 1970}

Shair Ahmad, Dynamical systems of characteristic $0^{+} \ldots \ldots \ldots \ldots \ldots$

Charles A. Akemann and Bernard Russo, Geometry of the unit sphere of a $C^{*}$-algebra and its dual............................ 575

Philip Bacon, The compactness of countably compact spaces ......... 587

Richard Blaine Barrar and Henry Loeb, On the continuity of the nonlinear Tschebyscheff operator ............................ 593

L. Carlitz, Factorization of a special polynomial over a finite field ....... 603

Joe Ebeling Cude, Compact integral domains .................... 615

Frank Rimi DeMeyer, On automorphisms of separable algebras. II . . . . . 621

James B. Derr, Generalized Sylow tower groups .................. 633

Raouf Doss, Some inclusions in multipliers ................... 643

Mary Rodriguez Embry, The numerical range of an operator........... 647

John Froese, Domain-perturbed problems for ordinary linear differential

operators..................................... 651

Zdeněk Frolík, Absolute Borel and Souslin sets ..................... 663

Ronald Owen Fulp, Tensor and torsion products of semigroups .......... 685

George Grätzer and J. Płonka, On the number of polynomials of an

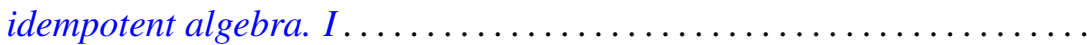

Newcomb Greenleaf and Walter Read, Positive holomorphic differentials on

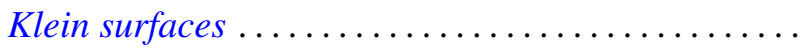

John Willard Heidel, Uniqueness, continuation, and nonoscillation for a second order nonlinear differential equation ................. 715

Leon A. Henkin, Extending Boolean operations................... 723

R. Hirshon, On hopfian groups .......................... 753

Melvin Hochster, Totally integrally closed rings and extremal spaces ..... 767

R. Mohanty and B. K. Ray, On the convergence of a trigonometric integral ..................................

Michael Rich, On a class of nodal algebras .................... 787

Emile B. Roth, Conjugate space representations of Banach spaces ........ 793

Rolf Schneider, On the projections of a convex polytope .............. 799

Bertram Manuel Schreiber, On the coset ring and strong Ditkin sets ...... 805

Edgar Lee Stout, Some remarks on varieties in polydiscs and bounded

holomorphic functions .........................

James Edward Ward, Two-groups and Jordan algebras . 